\title{
Herbicidas e danos físicos em tocos de teca para controle de brotos após o desbaste
}

\author{
Herbicides and physical damages in teak stumps to control of sprouts after thinning
}

\author{
Sidney Fernando Caldeira ${ }^{\mathrm{I}}$ Cibele Kotsubo da Cunha e Castro ${ }^{\text {II }}$
}

\section{RESUMO}

Tocos de árvores desbastadas de Tectona grandis apresentam rebrota intensa que compete com as árvores remanescentes. $O$ objetivo deste trabalho foi avaliar a eficácia da aplicação isolada ou combinada de diferentes concentrações dos herbicidas picloram e triclopyr, associados ou não à aplicação de danos físicos, no controle dessas brotações. Em um povoamento com quatro anos de idade, os tocos foram tratados imediatamente após o desbaste. Em outro povoamento com seis anos, foram tratadas as brotações presentes nos tocos desbastados no ano anterior. Foi registrada a porcentagem de tocos mortos, o número de brotações por toco e as respectivas alturas. No primeiro ensaio, a aplicação combinada de picloram a 0,48\% com triclopyr a 0,96\%, associada a 20 rachas com machado matou todos os tocos. No segundo ensaio, a maior eficácia, 21,7\%, foi registrada com roçada prévia das brotações e a aplicação de picloram a 0,96\%. Após o desbaste, a aplicação isolada de picloram ou combinada com triclopyr associada ou não aos danos físicos é eficiente para controlar os brotos de teca. A aplicação nas rebrotas de tocos desbastados no ano anterior apresenta alguma eficiência, mas com menor percentual de tocos mortos em relação à aplicação após o desbaste.

Palavras-chave: Tectona grandis, eliminação da rebrota, picloram, triclopyr.

\section{ABSTRACT}

The stumps of thinning trees of Tectona grandis L.f. present intense sprouts that compete with the remaining trees. The efficacy of the control of sprouts with the herbicides picloram and triclopyr, associated or no it applications of physical damages, were evaluated. Immediately after thinning, in plantation with four years old, the stumps were treated, and in other plantation, with age of six, the sprouts of stumps thinned in the previous year, were treated. The percentage of died stumps, the number of sprouts by stump and the respective heights were registered. In the first trial, the combined application of picloram at $0.48 \%$ with triclopyr at $0.96 \%$, associated a 20 cracks with axe killed all stumps. In the second trial, the best efficacy, $21.7 \%$, was obtained with the previous mowing of sprouts and application of picloram at 0.96\%. After thinning, the isolated application of picloram or combined with triclopyr, associates or not a physical damage is efficient to control sprouts of teak. The application in the sprouts of stumps thinned in the last year present any efficiency, with least percentage of dead stumps in relation to application after thinning.

Key words: Tectona grandis, elimination of sprouts, picloram, triclopyr.

\section{INTRODUÇÃO}

Os plantios comerciais de Tectona grandis L.f., teca, Lamiaceae iniciaram em 1970 em Cáceres, MT e em 2010 havia 65440 ha plantados no Brasil (ABRAF, 2011). Em 2007, Mato Grosso apresentava mais de 48500ha de teca para a produção de toras (SHIMIZU et al., 2007) e este tipo de produto exige rotações mais longas e a aplicação de desbastes. O desbaste diminui a densidade do povoamento e adéqua a ocupação espacial de copas e raízes, à medida que as árvores remanescentes se desenvolvem. Após o corte, os tocos de algumas espécies brotam e as brotações não eliminadas competirão com as árvores

IDepartamento de Engenharia Florestal, Faculdade de Engenharia Florestal (FENF), Universidade Federal de Mato Grosso (UFMT), Av. Fernando Correa da Costa, SN, Coxipó, 78068-000, Cuiabá, MT, Brasil. E-mail: sidcal@ufmt.br. Autor para correspondência. IIPrograma de Pós-graduação em Ciências Florestais e Ambientais, FENF, UFMT, Cuiabá, MT, Brasil. 
remanescentes. Segundo SOTO (1998), a teca apresenta esse comportamento com o desenvolvimento de um sub-bosque resistente e denso que, além da competição intraespecífica, ainda se torna um obstáculo físico para a aplicação de outras operações silviculturais.

Para controle das brotações, é utilizado o "rolo-faca", a remoção manual das brotações com o uso de foices, ou o controle químico com herbicidas, que é considerado um método eficaz e barato. Essa técnica vem sendo utilizada em plantios de espécies florestais, como em Eucalyptus populnea e $\boldsymbol{E}$. largiflorens com butil 2,4,5-T ou a mistura de picloram e 2,4,5-T com óleo diesel (ROBERTSON \& PEDERSEN, 1973; FERRELL et al., 2010; SCHALAU, 2011) ou o triclopyr isolado ou combinado com glifosato e metsulfuronmetil em $\boldsymbol{E}$. macarthurii, que possui alta capacidade de rebrota (LITTLE \& VAN DEN BERG, 2007). SOTO (1998) destacou que os métodos mais comuns de controle da rebrota de tocos de teca é o manual com a remoção da casca dos tocos para retirada das gemas e o uso de herbicidas. RODRIGUES \& ALMEIDA (2005) indicaram o triclopyr como opção de controle para plantas daninhas não controladas pelo glyphosate, além de apresentar rápida degradação no solo. Em solos com $\mathrm{pH}$ mais baixo, o seu grau de adsorção é mais elevado e a mobilidade é menor e, em condições de umidade e temperatura mais elevadas, a sua degradação é mais rápida. O uso de triclopyr e da combinação de 2,4-D + picloram controlaram Desmodium incanum e Zornia latifolia em gramados de Paspalum notatum (FREITAS et al., 2003). Em pastagens, foi obtido o controle de Memora peregrina, com a aplicação de picloram, após o corte com enxadão (NUNES, 1999); de Schinus terebintifolius e Eupatorium maximilianii com triclopyr e a combinação de 2,4-D + picloram ou de fluroxypyr + picloram, em pastagem de Melinis minutiflora (SANTOS et al., 2006)

O triclopyr é um herbicida com potencial de uso na eucaliptocultura, como é o caso de plantas de Eucalyptus urophylla que se recuperam quando expostas por deriva a este herbicida e que têm menor deriva em relação ao glyphosate, carfentrazone-ethyl e as suas misturas (TUFFI SANTOS et al., 2006). Segundo SOTO (1998), para o controle de brotações em tocos de Gmelina arborea e $\boldsymbol{T}$. grandis, o triclopyr foi mais eficiente em relação ao dicamba e ao glyphosate e mais barato e mais rápido que o controle manual.

O objetivo deste trabalho foi avaliar a eficácia no controle da brotação de tocos de teca, imediatamente após o desbaste e das rebrotas de tocos de árvores desbastadas no ano anterior, com a aplicação isolada ou combinada de diferentes concentrações de picloram e triclopyr, associada ou não à confecção de danos físicos aos tocos.

\section{MATERIAL E MÉTODOS}

O trabalho foi realizado na empresa Teca do Brasil Ltda., em Nossa Senhora do Livramento, MT. O solo da região é classificado como Planossolo Háplico Eutrófico (EMBRAPA, 2006) com teores médios de areia, silte e argila, respectivamente de 48,5\%; 11,5\% e $40 \%$. Em duas amostras de solo, coletadas em cada um dos povoamentos, não foi registrada a presença de Alumínio. As amostras do povoamento com seis anos de idade apresentaram 5,80 e 6,50 unidades de $\mathrm{pH}$, teores de Fósforo (P) de 6,5 e 7,6 $\mathrm{mg} \mathrm{dm}^{-3}$, de Potássio (K) 169 e $277 \mathrm{mg} \mathrm{dm}^{-3}$, de Cálcio + Magnésio $(\mathrm{Ca}+\mathrm{Mg})$ 7,5 e $9,7 \mathrm{cmol}_{\mathrm{c}} \mathrm{dm}^{-3}$, de Cálcio (Ca) 5,3 e 7,1 $\mathrm{cmol}_{\mathrm{c}} \mathrm{dm}^{-3} \mathrm{e}$ $3,3 \%$ e 3,5\% de matéria orgânica (MO). No povoamento com quatro anos, os resultados foram: 6,60 e 6,40 para o pH; 8,6 e 5,8mg dm${ }^{-3}$ para o P; 29 e $25 \mathrm{mg} \mathrm{dm}^{-3}$ para K; e valores iguais para $\mathrm{Ca}+\mathrm{Mg}$ de $2,4 \mathrm{cmol}_{\mathrm{c}} \mathrm{dm}^{-3}$, para o Ca de $1,3 \mathrm{cmol}_{\mathrm{c}} \mathrm{dm}^{-3}$ e $1,2 \%$ de MO.

Foram conduzidos dois ensaios, um em cada povoamento. O povoamento implantado em 1999 no espaçamento $3,0 \mathrm{mx} 3,0 \mathrm{~m}$ e desbastado aos cinco anos de idade apresentava os seguintes valores médios: densidade de 1083 árvores ha $^{-1}$, DAP de $12,3 \mathrm{~cm}$, altura total $(\mathrm{H})$ de $10,4 \mathrm{~m}$, área basal $(\mathrm{G})$ de $12,9875 \mathrm{~m}^{2} \mathrm{ha}^{-1} \mathrm{e}$ área transversal $(\bar{g})$ de $0,0120 \mathrm{~m}^{2}$ árvore $^{-1}$. O outro, implantado no espaçamento $3,0 \mathrm{mx} 2,0 \mathrm{~m}$, em 2001, foi desbastado aos quatro anos e apresentava 1597 árvores ha $^{-1}$, DAP de 8,1 cm, Hde 8,0m, G de 9,3675 $\mathrm{m}^{2}$ ha $^{-1} \mathrm{e} \bar{g} \mathrm{de}$ $0,0059 \mathrm{~m}^{2}$ árvore $\mathrm{e}^{-1}$.

O primeiro ensaio foi conduzido em maio de 2005 no povoamento com quatro anos de idade e foram utilizados tocos de 100 árvores desbastadas por tratamento, divididos em quatro repetições, em blocos casualizados. Após o desbaste, os tratamentos consistiram da aplicação de um tipo de dano físico seguido da pulverização de diferentes dosagens em porcentagem dos herbicidas picloram $(\% \mathrm{P})$ e triclopyr (\% T), isolados ou combinados. Os danos físicos de quatro rachas (4R) ou de cerca de 20 rachas (20R) foram efetuados com um machado, além de três furos com cerca de $10 \mathrm{~cm}$ de profundidade $(3 \mathrm{~F})$ foram efetuados com o uso de broca de $1 / 2$ polegada e furadora motorizada (Tabela 1). O segundo ensaio foi conduzido em setembro de 2005 no povoamento com seis anos de idade, desbastado no ano anterior. Os tratamentos foram aplicados da forma descrita anteriormente e o dano físico foi a roçada manual com foice das brotações (RB) e a aplicação isolada ou combinada dos mesmos herbicidas (Tabela 2). 
Tabela 1 - Tratamentos aplicados em tocos de Tectona grandis com quatro anos de idade, imediatamente após o desbaste. Nossa Senhora do Livramento, MT, maio, 2005.

\begin{tabular}{lccc}
\hline Tratamento & Picloram $(\%)$ & Triclopyr $(\%)$ & Dano físico aplicado \\
\hline $0,96 \% \mathrm{P}$ & 0,96 & --- & Sem dano físico \\
$0,72 \% \mathrm{P}$ & 0,72 & --- & Sem dano físico \\
$0,6 \% \mathrm{P}$ & 0,6 & --- & Sem dano físico \\
$0,96 \% \mathrm{P}$ e $4 \mathrm{R}$ & 0,96 & --- & 4 rachas próximas ao câmbio \\
$0,72 \% \mathrm{P}$ e $4 \mathrm{R}$ & 0,72 & --- & 4 rachas próximas ao câmbio \\
$0,6 \% \mathrm{P}$ e $4 \mathrm{R}$ & 0,6 & --- & 4 rachas próximas ao câmbio \\
$0,96 \% \mathrm{P}$ e 3F & 0,96 & --- & 3 furos de $1 / 2$ " próximos ao câmbio \\
$0,72 \% \mathrm{P}$ e 3F $3 \mathrm{~F}$ & --- & 3 furos de $1 / 2$ " próximos ao câmbio \\
$0,6 \% \mathrm{P}$ e 3F & 0,72 & --- & 3 furos de $1 / 2$ " próximos ao câmbio \\
$0,48 \% \mathrm{P}+0,96 \% \mathrm{~T}$ e 20R & 0,6 & 0,96 & 20 rachas distribuídas no toco \\
$0,36 \% \mathrm{P}+0,72 \% \mathrm{~T}$ e 3F & 0,48 & 0,72 & 3 furos de $1 / 2$ " próximos ao câmbio \\
$0,36 \% \mathrm{P}+0,72 \% \mathrm{~T}$ e 20R & 0,36 & 0,72 & 20 rachas distribuídas no toco \\
Testemunha & 0,36 & --- & Sem operação \\
\hline
\end{tabular}

Os ensaios foram avaliados, respectivamente, em fevereiro e em junho de 2006. A eficácia foi determinada pela porcentagem de tocos mortos (TM), a média do número de brotações (NB) e a respectiva altura das brotações (HB) dos tocos sobreviventes. Os dados foram transformados em $\sqrt{\mathrm{y}+1}$ e, após a análise de variância, as médias foram comparadas pelo teste de Scott-Knott a 5\% de probabilidade, com o programa Sisvar 5.0 (FERREIRA, 2008). Também foi registrado o tempo gasto para a execução dos tratamentos e calculada a média do número de tocos tratados por hora (NTT), para comparação do rendimento operacional.

\section{RESULTADOS E DISCUSSÃO}

A eficácia de $100 \%$ de tocos mortos foi obtida com a aplicação de $0,48 \% \mathrm{P}+0,96 \% \mathrm{~T}$ associada a 20 rachas. Contudo, esse tratamento não diferiu de outros seis tratamentos, sendo que os maiores valores absolutos para esta variável foram com a combinação dos produtos, $95 \%$ e $90 \%$, e com as maiores concentrações apenas de picloram, associado a algum tipo de ferimento no toco, 78,4\% a 88,3\% (Tabela 3). A aplicação de $0,96 \%$ P, sem dano físico, $85 \%$, não diferiu dos tratamentos mencionados e indica a possibilidade de sua aplicação isolada, o que possibilitaria tratar até 130 tocos por hora, enquanto a execução das 20 rachas ou dos três furos permite o tratamento de 84 e 92 tocos por hora, respectivamente.

Controles semelhantes foram registrados com picloram a $4 \%$ para $A$. farnesiana e $M$. pteridofita (CARMONA et al., 2001) para A. altissima, com a aplicação de triclopyr combinado com picloram ou imazapyr (BURCH \& ZEDAKER, 2003) e para $\boldsymbol{A}$. dealbata, A. melanoxylon e E. globulus, após o corte e pincelamento com triclopyr, 95 a $99 \%$ (SANTOS \& MONTEIRO, 2007). A menor eficácia também foi

Tabela 2 - Tratamentos em brotações de tocos de Tectona grandis com seis anos de idade, desbastados no ano anterior. Nossa Senhora do Livramento, MT, setembro, 2005.

\begin{tabular}{lccl}
\hline Tratamento & Picloram $(\%)$ & Triclopyr $(\%)$ & Operação aplicada \\
\hline $0,96 \%$ P e RB & 0,96 & -- & Roçada das brotações \\
$0,72 \%$ P RB & 0,72 & --- & Roçada das brotações \\
$0,6 \%$ P RB & 0,6 & --- & Roçada das brotações \\
$0,48 \%$ P+0,96\%T e RB & 0,48 & 0,96 & Roçada das brotações \\
$0,96 \%$ P & 0,96 & --- & Sem operação \\
$0,72 \% \mathrm{P}$ & 0,72 & --- & Sem operação \\
$0,6 \% \mathrm{P}$ & 0,6 & --- & Sem operação \\
$0,48 \% \mathrm{P}+0,96 \% \mathrm{~T}$ & 0,48 & 0,96 & Sem operação \\
Testemunha & --- & --- & Sem operação \\
\hline
\end{tabular}


Tabela 3 - Tocos mortos (TM), número (NB) e altura (HB) das brotações e o número de tocos tratados por hora (NTT) com picloram (\%P) e triclopyr (\%T), associados ou não aos danos físicos de quatro rachas (4R), 20 rachas (20R) ou três furos (3F), após o desbaste de um povoamento de Tectona grandis com quatro anos de idade.

\begin{tabular}{|c|c|c|c|c|}
\hline Tratamento & $\mathrm{TM}(\%)$ & NB (un) & $\mathrm{HB}(\mathrm{cm})$ & $\operatorname{NTT}\left(\mathrm{N} \mathrm{h}^{-1}\right)$ \\
\hline $0,48 \% \mathrm{P}+0,96 \% \mathrm{~T}$ e $20 \mathrm{R}$ & $100,0 \mathrm{~A}$ & $0,0 \mathrm{~A}$ & $0,0 \mathrm{~A}$ & 92 \\
\hline $0,36 \% \mathrm{P}+0,72 \% \mathrm{~T}$ e $3 \mathrm{~F}$ & $95,0 \mathrm{~A}$ & $0,8 \mathrm{~A}$ & $72,5 \mathrm{~A}$ & 90 \\
\hline $0,36 \% \mathrm{P}+0,72 \% \mathrm{~T}$ e $20 \mathrm{R}$ & $90,0 \mathrm{~A}$ & $1,1 \mathrm{~B}$ & $154,3 \mathrm{~B}$ & 84 \\
\hline $0,72 \% \mathrm{P} \mathrm{e} 4 \mathrm{R}$ & $88,3 \mathrm{~A}$ & $1,8 \mathrm{~B}$ & $154,5 \mathrm{~B}$ & 137 \\
\hline $0,96 \%$ P e $3 F$ & $86,7 \mathrm{~A}$ & $1,5 \mathrm{~B}$ & $123,0 \mathrm{~B}$ & 98 \\
\hline $0,96 \% \mathrm{P}$ & $85,0 \mathrm{~A}$ & $1,3 \mathrm{~B}$ & $184,0 \mathrm{~B}$ & 130 \\
\hline $0,6 \% \mathrm{P}$ e $4 \mathrm{R}$ & $78,4 \mathrm{~A}$ & $1,2 \mathrm{~B}$ & $199,5 \mathrm{~B}$ & 151 \\
\hline $0,96 \% \mathrm{P} \mathrm{e} 4 \mathrm{R}$ & $73,4 \mathrm{~B}$ & $2,6 \mathrm{C}$ & $199,0 \mathrm{~B}$ & 116 \\
\hline $0,72 \%$ P e $3 F$ & $73,3 \mathrm{~B}$ & $1,4 \mathrm{~B}$ & $205,3 \mathrm{~B}$ & 99 \\
\hline $0,6 \% \mathrm{P}$ e $3 \mathrm{~F}$ & $61,7 \mathrm{~B}$ & $1,7 \mathrm{~B}$ & $193,3 \mathrm{~B}$ & 113 \\
\hline $0,72 \% \mathrm{P}$ & $53,4 \mathrm{~B}$ & $1,7 \mathrm{~B}$ & $238,8 \mathrm{~B}$ & 142 \\
\hline $0,6 \% \mathrm{P}$ & $31,7 \mathrm{C}$ & $2,9 \mathrm{C}$ & 243,0 B & 135 \\
\hline Testemunha & $0,0 \mathrm{D}$ & $4,7 \mathrm{D}$ & $406,5 \mathrm{C}$ & 0 \\
\hline CV (\%) & 9,24 & 15,40 & 12,53 & --- \\
\hline
\end{tabular}

Médias com a mesma letra são iguais pelo teste de Scott-Knott $(\mathrm{P}>0,05)$ com os dados transformados em $\sqrt{ }$ y+1. As médias na tabela são os valores originais.

registrada com a utilização de concentrações mais baixas, por NUNES (2001) no controle parcial de $\boldsymbol{M}$. peregrina em pastagem, 66,7\%, com a aplicação de triclopyr a $2 \%$ e de $82,3 \%$ com o uso de picloram a $1,5 \%$, contudo com a aplicação de $4 \%, 6 \%$ e $20 \%$ de triclopyr, associado com picloram, a eficácia foi superior a 90\%. É possível verificar que esses trabalhos utilizaram maiores concentrações em relação àquelas que foram testadas nos tocos de teca.

Quanto ao número e à altura das brotações dos tocos sobreviventes, estas variáveis apresentaram resultado bastante semelhante, quanto à ordem decrescente em eficácia, em relação à porcentagem de tocos mortos. Ainda assim, a maior eficácia parece estar relacionada à maior concentração absoluta dos ingredientes ativos utilizados $(1,44 \%$ e $1,08 \%)$, do que propriamente da combinação dos produtos ou aos danos físicos que foram efetuados.

Após nove meses, a testemunha apresentou $100 \%$ de tocos vivos e estes apresentaram o maior número de brotações, 4,7, e o maior desenvolvimento em altura, $406,5 \mathrm{~cm}$, superiores a todos os outros tratamentos, o que indica a competição intraespecífica, sendo necessário aplicar algum tipo de controle. A opção de diminuir a densidade do desbaste pode não ser uma boa estratégia, pois, segundo CHOWDHURY et al. (2008), diferentes regimes de abertura da copa não afetam a taxa de sobrevivência e o número de brotações. $\mathrm{O}$ toco remanescente deve ser o menor possível, pois o número de brotações é maior para tocos com 10 a $20 \mathrm{~cm}$ de altura, apresentam correlação com o diâmetro da árvore e da cepa e que as medições devem ser feitas após seis meses, pois o diâmetro e a altura das brotações não diferem até esse período, mas diferem aos 12 e 18 meses.

$\mathrm{O}$ tratamento sem qualquer dano físico ao toco permitiu o tratamento de 135 tocos por hora com um pulverizador costal manual. Para efetuar as quatro rachas, foi necessário o envolvimento de um segundo operador e o rendimento da equipe foi de 135 tocos por hora. Contudo, para fazer os três furos com a broca motorizada, o rendimento foi de 100 tocos por hora e, para fazer as 20 rachas, apenas de 88 (Tabela 3). O tempo gasto pelo operador para danificar os tocos sempre foi superior ao do operador do pulverizador, exceto para as quatro rachas, que foi semelhante, pela sincronia operacional que foi observada.

Alguns tocos sobreviventes apresentaram sintomas de fitotoxidez nas brotações, com encrespamento e clorose parcial de folhas jovens (Figura 1A), o encrespamento da ponta de folhas adultas (Figura 1B), além de formação incompleta da ponta e da borda de folhas, com clorose parcial distribuída no limbo foliar (Figura 1C). Sintomas semelhantes ocorrem em eucalipto após a aplicação do fungicida captafol (FERREIRA \& MILANI, 2002). Contudo, SOTO (1998), além de obter eficácia no controle da rebrota dos tocos de $\boldsymbol{T}$. grandis e de $\boldsymbol{G}$ arborea, com o triclopyr, não registrou sintomas de fitotoxidez com esse produto, com o dicamba e o glyphosate. 


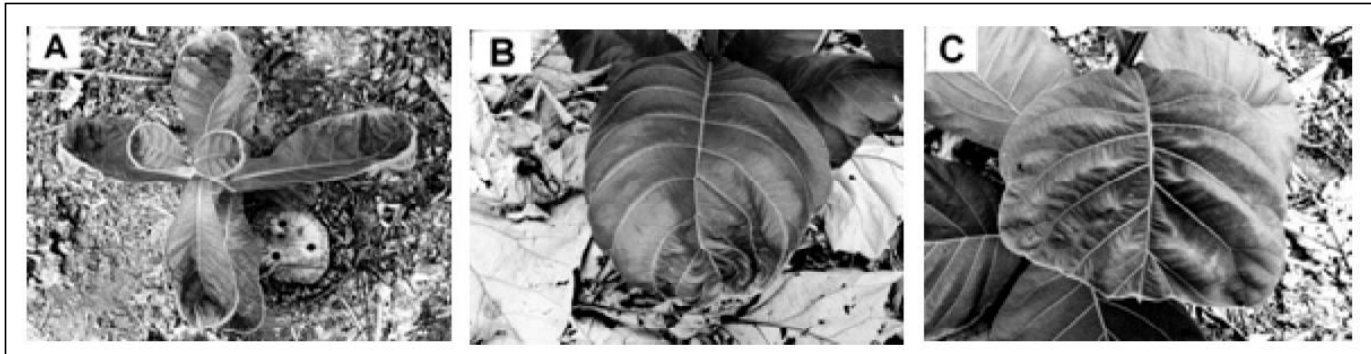

Figura 1 - Fitotoxidez em brotações de tocos de Tectona grandis tratados com picloram e triclopyr após o desbaste: (A) encrespamento com clorose parcial em folhas jovens e (B) em folha adulta e (C) folha com a borda incompleta e mal formada.

Os tratamentos efetuados nas brotações de tocos de árvores desbastadas no ano anterior apresentaram menores porcentagens de tocos mortos (Tabela 4), comparados com a aplicação nos tocos após o desbaste (Tabela 3), em que o menor valor foi de $31,7 \%$ e os tocos sobreviventes continham 2,9 brotações com $243,0 \mathrm{~cm}$ de altura. No segundo ensaio, o melhor tratamento $(0,96 \% \mathrm{P}+\mathrm{RB})$ resultou em $21,7 \%$ de tocos mortos e os tocos sobreviventes com 6,2 brotações, mas com $42,0 \mathrm{~cm}$ de altura (Tabela 4). Os melhores tratamentos foram aqueles em que as diferentes concentrações dos produtos foram aplicadas após a roçada das brotações com 16,7 a 21,7\% de tocos mortos. Quanto à ordem decrescente em eficácia, o número e a altura das brotações por toco apresentaram bastante variação em relação à porcentagem de tocos mortos.

O picloram e o triclopyr são absorvidos por via foliar e radicular e a aplicação do picloram na região do câmbio (floema), logo após o corte, favorece a sua translocação através do plasmalema até as raízes, enquanto o triclopyr necessita de quatro horas para ser absorvido pelas folhas, ser translocado e acumulado nos tecidos meristemáticos (NUNES, 2001). Isso explicaria o melhor resultado obtido com a aplicação após a roçada das brotações. Contudo, a eficácia foi menor, pois a área de translocação obtida pelo corte das brotações é inferior à área do toco após o desbaste.

Neste ensaio, ocorreu morte natural de 1,7\% dos tocos da testemunha, com diâmetro menor, provavelmente em decorrência da maior competição com as árvores remanescentes. Os tocos sobreviventes apresentaram até menor número de brotações do que outros três tratamentos, contudo a altura das brotações foi de $401 \mathrm{~cm}$, enquanto para os tratamentos, o maior valor foi de $182 \mathrm{~cm}(0,72 \% \mathrm{P})$. Somente a altura das brotações da testemunha foi superior a dos tratamentos (Tabela 4). As testemunhas dos dois ensaios apresentaram valores numericamente semelhantes (Tabelas 3 e 4).

Somente para a aplicação dos produtos nas brotações dos tocos que haviam sido desbastados no ano anterior, o operador apresentou um rendimento de 95 tocos por hora. Para efetuar a roçada das brotações e a aplicação dos produtos, o rendimento operacional

Tabela 4 - Tocos mortos (TM), número (NB) e altura (HB) das brotações e o número de tocos tratados por hora (NTT) com picloram (\%P) e triclopyr $(\% \mathrm{~T})$, associados ou não à roçada das brotações $(\mathrm{RB})$, de tocos das árvores desbastadas no ano anterior de um povoamento de Tectona grandis com seis anos de idade.

\begin{tabular}{|c|c|c|c|c|}
\hline Tratamento & $\mathrm{TM}(\%)$ & NB (un) & $\mathrm{HB}(\mathrm{cm})$ & NTT $\left(\mathrm{N} \mathrm{h}^{-1}\right)$ \\
\hline $0,96 \%$ P e RB & $21,7 \mathrm{~A}$ & $6,2 \mathrm{~B}$ & $42,0 \mathrm{~A}$ & 68 \\
\hline $0,48 \% \mathrm{P}+0,96 \% \mathrm{~T}$ e $\mathrm{RB}$ & $16,7 \mathrm{~A}$ & $3,7 \mathrm{~A}$ & $39,8 \mathrm{~A}$ & 71 \\
\hline $0,72 \% \mathrm{P}$ e $\mathrm{RB}$ & $16,7 \mathrm{~A}$ & $5,7 \mathrm{~B}$ & $41,0 \mathrm{~A}$ & 78 \\
\hline $0,72 \% \mathrm{P}$ & $11,7 \mathrm{~A}$ & $4,4 \mathrm{~A}$ & $182,0 \mathrm{C}$ & 96 \\
\hline $0,6 \% \mathrm{P}$ e $\mathrm{RB}$ & $11,7 \mathrm{~A}$ & $6,0 \mathrm{~B}$ & $38,5 \mathrm{~A}$ & 117 \\
\hline $0,48 \% \mathrm{P}+0,96 \% \mathrm{~T}$ & $8,4 \mathrm{~A}$ & $4,0 \mathrm{~A}$ & $155,3 \mathrm{~B}$ & 89 \\
\hline $0,96 \% \mathrm{P}$ & $3,4 \mathrm{~B}$ & $7,8 \mathrm{C}$ & $149,0 \mathrm{~B}$ & 94 \\
\hline $0,6 \% \mathrm{P}$ & $1,7 \mathrm{~B}$ & $4,0 \mathrm{~A}$ & $161,3 \mathrm{~B}$ & 99 \\
\hline Testemunha & $1,7 \mathrm{~B}$ & $5,9 \mathrm{~B}$ & $401,0 \mathrm{D}$ & 0 \\
\hline $\mathrm{CV}(\%)$ & 49,46 & 7,37 & 2,56 & --- \\
\hline
\end{tabular}

Médias com a mesma letra são iguais pelo teste de Scott-Knott $(\mathrm{P}>0,05)$ com os dados transformados em $\sqrt{ }$ y+1. As médias na tabela são os valores originais. 
da equipe foi reduzido para 83 tocos por hora (Tabela 4), mas essa operação, associada às maiores concentrações do picloram ou a sua associação com triclopyr, aumentou a eficácia na mortalidade das brotações dos tocos.

\section{CONCLUSÃO}

Aplicações nos tocos de teca, imediatamente após o desbaste, de picloram + triclopyr associadas a danos físicos ou apenas de picloram associadas ou não a danos físicos, são eficientes para controlar as brotações e a eficácia diminui com a redução da concentração.

Aplicações nas brotações de tocos desbastados no ano anterior, de picloram ou sua associação com triclopyr com ou sem danos físicos, apresenta alguma eficiência, mas com menor mortalidade em relação ao tratamento efetuado imediatamente após o desbaste.

A execução de quatro rachas com a pulverização dos produtos não diminui o número de tocos tratados em relação à operação de pulverização, enquanto a perfuração e a execução das 20 rachas diminuem o número de tocos tratados por hora, além de exigirem a presença de um segundo operador.

\section{AGRADECIMENTO} deste trabalho.

À empresa Teca do Brasil, pelo apoio na realização

\section{REFERÊNCIAS}

ABRAF. Anuário estatístico da ABRAF 2011 ano base 2010. Brasília: ABRAF, 2011. 130p. Disponível em: <http:/ /www.abraflor.org.br/estatisticas/ABRAF11/ABRAF11-BR.pdf>. Acesso em: 02 jun. 2010.

BURCH, P.L.; ZEDAKER, S.M. Removing the invasive tree Ailanthus altissima and restoring natural cover. Journal of Arboriculture, Illinois, v.29, n.1, p.18-24, 2003. Disponível em: <http://www.treelink.org/joa/2003/jan/03Burch.pdf >. Acesso em: 08 mar. 2010.

CARMONA, R. et al. Controle de Acacia farnesiana e de Mimosa pteridofita em pastagem. Pesquisa agropecuária Brasileira, Brasília, v.36, n.10, p.1301-1307, 2001. Disponível em: <http://www.scielo.br/scielo.php?pid=S0100$204 \mathrm{X} 2001001000013 \&$ script $=$ sci arttext $>$. Acesso em: 22 abr. 2007. doi: 10.1590/S0100-204X2001001000013.

CHOWDHURY, M.D.Q. et al. Growth performance of teak (Tectona grandis Linn. f.) coppice under different regimes of canopy opening. Tropical Ecology, Varanasi, v.49, n.2, p.245250, 2008. Disponível em: <http://www.tropecol.com/pdf/open/ PDF_49_2/15\%20Chowdhury.pdf $>$. Acesso em: 13 jan. 2009.
EMBRAPA, Centro nacional de Pesquisa de Solos. Sistema brasileiro de classificação de solos. 2.ed. Rio de Janeiro, 2006. 306p. Disponível em: <http://www.cnps.embrapa.br/ sibcs/download/ata10.pdf>. Acesso em: 04 abr. 2007.

FERREIRA, D.F. SISVAR: um programa para análise e ensino da estatística. Revista Científica Symposium, Lavras, v.6, n.2, p.36-41, 2008. Disponível em: <http://www.dex.ufla.br/ $\sim$ danielff/softwares.htm>. Acesso em: 13 set. 2009.

FERREIRA, F.A.; MILANI, D. Diagnose visual e controle das doenças abióticas e bióticas do eucalipto no Brasil. Mogi Guaçu, SP: International Paper, 2002. 98p.

FERRELL, J. et al. Herbicide application techniques for woody plant control. Gainesville, FL: Agronomy Department, IFAS Extension, University of Florida, 2010. 4p. (Documento SS-AGR-260). Disponível em: <http://edis.ifas.ufl.edu/pdffiles/ AG/AG24500.pdf>. Acesso em: 25 jan. 2012.

FREITAS, F.C.L. et al. Eficiência do triclopyr no controle de plantas daninhas em gramado (Paspalum notatum). Planta Daninha, Viçosa, v.21, n.1, p.159-164, 2003. Disponível em: <http://www.scielo.br/pdf/pd/v21n1/a20v21n1.pdf >. Acesso em: 06 jan. 2005.

LITTLE, K.M.; VAN DEN BERG, G.J. Comparison of different herbicides for single stem Eucalyptus macarthurii cut stump control. Journal of Tropical Forest Science, Malaysia, v.19, n. 1, p.13-17, 2007

NUNES, S.G. Ciganinha, Memora peregrina (Miers) Sandw, nova planta invasora de pastagem. Campo Grande: EMBRAPA Gado de Corte, 1999. 3p. (Embrapa Gado de Corte. Embrapa Gado de Corte Divulga n.35). Disponível em: <http://www.cnpgc.embrapa.br/publicacoes/divulga/ GCD35.html>. Acesso em: 22 fev. 2010.

NUNES, S.G. Controle de plantas invasoras em pastagens cultivadas nos Cerrados. Campo Grande: EMBRAPA Gado de Corte, 2001. 36p. (Embrapa Gado de Corte. Documentos, 117). Disponível em: <http://www.cnpgc.embrapa.br/publicacoes/ doc/doc117/05metodos.html $>$. Acesso em: 22 fev. 2010.

ROBERTSON, J.A.; PEDERSEN, C.H. Chemical control of eucalypt regrowth. Tropical Grasslands, v.7, n.2, p.223$227,1973$.

RODRIGUES, B.N.; ALMEIDA, F.S. Guia de herbicidas. 5.ed. Londrina: Grafmarke, 2005. 591p.

SANTOS, A.R.; MONTEIRO, A. Controlo de invasoras lenhosas no Parque Ecológico do Funchal. Silva Lusitana, Lisboa, v.15, n.2, p.249-255, 2007. Disponível em: <http://www.scielo.oces.mctes.pt/ scielo.php?pid=S0870-63522007000200007\&script=sci_arttext $>$. Acesso em: 08 jun. 2009.

SANTOS, M.V. et al. Eficácia e persistência no solo de herbicidas utilizados em pastagem. Planta Daninha, Viçosa, v.24, n.2, p.391398, 2006. Disponível em: <http://www.scielo.br/scielo.php?pid= S0100-83582006000200024\&script=sci_arttext $>$. Acesso em: 13 fev. 2010. doi: 10.1590/S0100-83582006000200024.

SCHALAU, J. Cut stump application of herbicides to manage woody vegetation. Tucson: The University of 
Arizona College of Agriculture and Life Sciences, 2011. 2p. Disponível em: 〈http://ag.arizona.edu/pubs/garden/az1401.pdf〉. Acesso em: 25 jan. 2012.

SHIMIZU, J.Y. et al. Diagnóstico das plantações florestais em Mato Grosso. Cuiabá, MT: Central de Texto, 2007. 63p.

SOTO, M.D. Manejo de tocones (Gmelina arborea) y Teca (Tectona grandis) posterior al raleo. 1998. [s.p.]. Tesis (Lic. Ig. Agr.) CATIE, Guácimo, Costa Rica. Disponível em: <http://orton.catie.ac.cr/
cgi-bin/wxis.exe/?IsisScript=TESISUM.xis\&method=post\&formato= $2 \&$ cantidad=1\&expresion=mfn=000991 $>$. Acesso em: 16 jun. 2009.

TUFFI SANTOS, L.D. et al. Intoxicação de eucalipto submetido à deriva simulada de diferentes herbicidas. Planta Daninha, Viçosa, v.24, n.3, p.521-526, 2006. Disponível em: <http:// www.scielo.br/scielo.php? script=sci_arttext\&pid=S010083582006000300014>. Acesso em: 22 maio, 2008. doi: 10.1590/S0100-83582006000300014.

Ciência Rural, v.42, n.10, out, 2012. 